\title{
ACUTE EFFECTS OF RESISTANCE AND AEROBIC EXERCISES WITH BLOOD FLOW RESTRICTION ON BLOOD PRESSURE IN ELDERLY
}

\author{
Lucas C. Santos*, Amanda V. Sardeli, Marina Lívia V. Ferreira, Claúdia R. Cavaglieri, Mara Patrícia T. Chacon- \\ Mikahil.
}

\begin{abstract}
The aim was to compare the acute effect of resistance(RE) and aerobic exercise(AE) with blood flow restriction(BFR) on systolic(SBP) and diastolic blood pressure(DBP) in healthy elderly. By mixed model for repeated measures and Bonferroni post hoc, we observed lower values in the end of $A E$ compared to RE(SBP-AE:148 $\pm 19 ; \mathrm{RE}: 215 \pm 24$; $D B P-A E: 75 \pm 11$; $\mathrm{RE}: 120 \pm 16 \mathrm{mmHg}$ ) as well the DBP at 30 minutes after exercises(AE:119 $\pm 19 ; \mathrm{RE}: 128 \pm 14 \mathrm{mmHg})$. We concluded AEBFR was safer than REBFR in this population.
\end{abstract}

Key-word:

Aerobic exercise, Resistance exercise, blood flow restriction.

\section{Introduction}

The blood flow restriction (BFR) has been associated to low-intensity exercises providing similar benefits to conventional exercise protocols such a aerobic exercise $(A E)$ and resistance exercise (RE). AE and RE with BFR are able to increase strenght and muscle mass what could be a good choice for exemple for elderly people, however it is not clearly which of these protocols can be safer for the cardiovascular system. It might be interesting to compare the response of the systolic blood pressure (SBP) and diastolic blood pressure (DBP), during and after theseprotocols especially in elderly. Thus the objective of this study was to compare the effects of aerobic exercise with blood flow restriction (AE-BFR), and resistance exercise with blood flow restriction (RE-BFR) on the SBP and DBP.

\section{Results and Discussion}

After screening and clinical tests, 17 older adults were included in the study ( 9 women, 8 men, $63.5 \pm 4.2$ years, $65.6 \pm 12.8 \mathrm{~kg})$. After familiarization with protocols, maximum strength test (1RM) and aerobic fitness test (VO'max.) they were cross-over randomized for start with AE-BFR or RE-BFR experimental protocols. The experimental protocols AE-BFR (40\% VO2max, $20 \mathrm{~min}$ walk on a treadmill with $50 \%$ of BFR.) and RE-BFR $(30 \%$ $1 \mathrm{RM}, 1$ series 30 repetitions +3 sets of 15 repetitions with $50 \%$ of blood flow restriction) were separated by a minimum of $72 \mathrm{~h}$. SBP and DBP were collected through Finometer-PRO device (Finapres Medical Systems). We performed mixed models for repeated measures analyses with Bonferroni post hoc in SPSS. SBP and DBP were similar in the baseline. Both protocols increased SBP and DBP in the end of exercise, with RE-BFR causing higher peaks. After 15 seconds of the end of both exercise protocols, SBP and DBP returned to baseline levels, however SBP still higher for RE-BFR. PAD after 30 minutes of effort remained higher than AE-BFR. In addition, we also found higher mean blood preassure, heart rate and cardiac output after RE-BFR than AE-BF R.

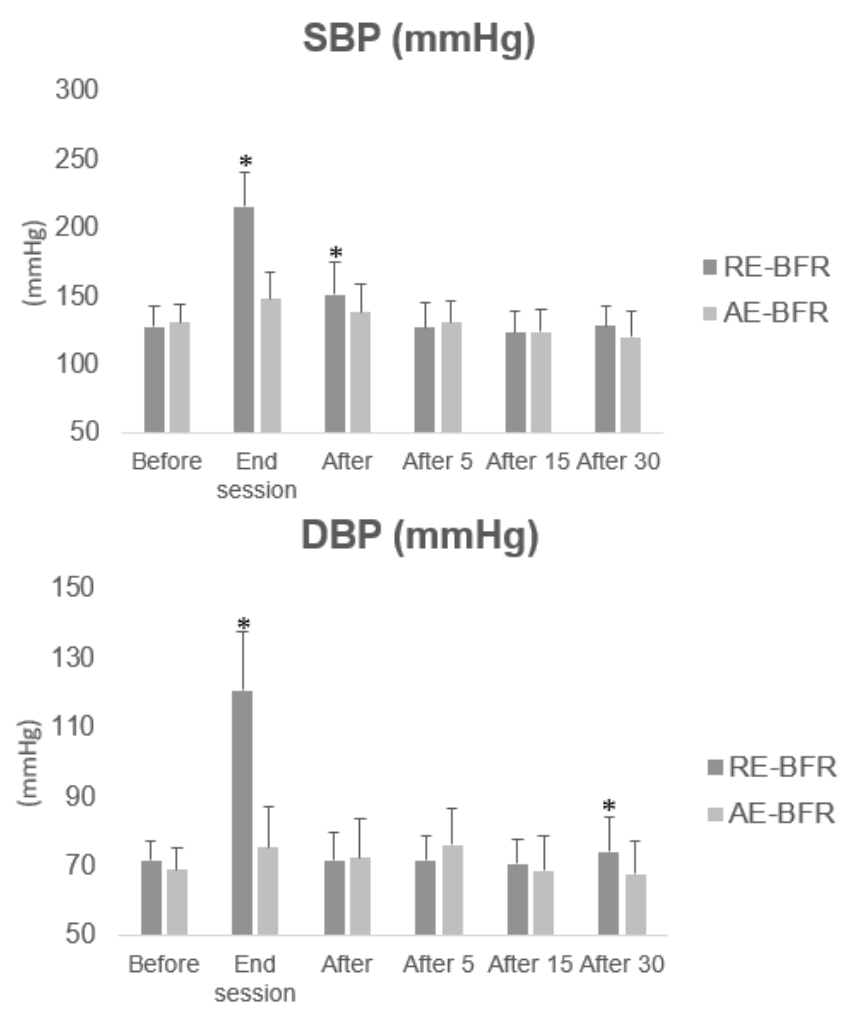

Figura 1. Values of SBP and DBP in mean and standard deviation, after the aerobic exercise with blood flow restriction (AE-BFR) and the resistance exercise with blood flow restriction (RE-BFR).. *Means $\mathrm{P}<0.05$ compared with AE-RFS.

\section{Conclusions}

Although both BFR exercise protocols have allowed a fast return of SBP and DBP for baseline levels, RE-BFR presented higher increases, suggesting greater cardiovascular security for EA-BFR.

\section{Acknowledgement}

We thanks FISEX, FEF-UNICAMP and LABFEF who supported us in this research.

ABE, T.; SAKAMAKI, M.; FUJITA, S.; OZAKI, H.; SUGAYA, M.; SATO, T.; NAKAJIMA, T.; Effects low-intesity walk training with restricted leg blood flow on muscle strength and aerobic capacity in older adults. Journal of geriatric physical therapy. v.33, n.1, p.34-40, 2010. 